\title{
Article
}

\section{Orbital motions of a solar sail around the L2 Earth-Moon libration point}

Gong, Shengping and Simo, Jules

Available at https://clok.uclan.ac.uk/12607/

Gong, Shengping and Simo, Jules orcid iconORCID: 0000-0002-1489-5920

(2014) Orbital motions of a solar sail around the L2 Earth-Moon libration point. Journal of Guidance, Control and Dynamics, 37 (4). pp. 1349-1356. ISSN 07315090

It is advisable to refer to the publisher's version if you intend to cite from the work.

http://dx.doi.org/10.2514/1.G000063

For more information about UCLan's research in this area go to

http://www.uclan.ac.uk/researchgroups/ and search for < name of research Group>.

For information about Research generally at UCLan please go to http://www.uclan.ac.uk/research/

All outputs in CLoK are protected by Intellectual Property Rights law, including Copyright law. Copyright, IPR and Moral Rights for the works on this site are retained by the individual authors and/or other copyright owners. Terms and conditions for use of this material are defined in the policies page. 


\title{
Orbital Motions of a Solar Sail around the $\mathbf{L}_{2}$ Earth-Moon Libration Point
}

\author{
Shengping Gong ${ }^{*}$ and Junfeng $\mathrm{Li}^{\dagger}$ \\ Tsinghua University, Beijing, 100084, CHINA \\ People's Republic of China \\ and
}

Jules Simo

University of Strathclyde, Glasgow, Scotland G1 1XJ, United Kingdom

\section{Nomenclature}

$\mathrm{A}=$ total area of the sail film

$\mathrm{A}_{\max }=$ area of sail film equipped with reflectivity control device

$\mathrm{A}_{1}=$ area of sail in state two

$\mathbf{a}_{\mathrm{S}}=$ solar radiation pressure acceleration exerted on the solar sail

$\mathrm{a}_{\mathrm{x}}, \mathrm{a}_{\mathrm{y}}, \mathrm{a}_{\mathrm{z}}=$ components of the solar radiation pressure acceleration in $\mathrm{x}, \mathrm{y}, \mathrm{z}$ direction, respectively

$\mathrm{i}=$ angle between the ecliptic plane and Moon's orbital plane

$\mathrm{m}=$ total mass of the solar sail

$\mathbf{n}=$ unit vector along the sail normal direction

$\mathrm{P}=$ solar radiation pressure

$\mathbf{r}=$ position vector of the sail with respect to the barycenter of the Earth and Moon

$\mathbf{r}_{\mathrm{S}}=$ unit vector along the sunlight direction in the ecliptic plane

$\mathrm{r}_{1}=$ distance from the sail to the Earth

$r_{2}=$ distance from the sail to the Moon

$\mathbf{S}=$ sliding surface

$\mathrm{u}=$ reflectivity rate of the sail

$\Gamma=$ weight matrix between the position error and velocity error in sliding surface

$\theta=$ angle measured from the Earth-Moon line to the $\mathrm{x}$ axis

\footnotetext{
*Associate professor, School of Aerospace; gongsp@tsinghua.edu.cn.

$\dagger$ Professor, School of Aerospace; lijunf@tsinghua.edu.cn.

$\$$ Academic Visitor, Advanced Space Concepts Laboratory, Department of Mechanical and Aerospace Engineering, University of Strathclyde; jules.simo@strath.ac.uk.
} 
$\kappa=$ dimensionless characteristic acceleration of the solar sail

$\mu=$ ratio of the Moon's mass to the total mass of the Earth and Moon

$\rho_{\mathrm{S}}=$ specular reflectivity coefficient

$\sigma=$ positive diagonal matrix for the sliding mode control

$\varphi=$ angle measured from the sunlight direction to the $\mathrm{x}$ axis

$\omega_{\mathrm{E}}=$ angular velocity of the Earth rotating around the Sun

$\omega_{\mathrm{M}}=$ angular velocity of the Moon rotating around the Earth

\section{Introduction}

Artificial Lagrange points and periodic orbits around them in the restricted three-body problem have attracted a great deal of attention. Solar sails provide new families of libration points inside regions connected to the classical libration points [1]. Similar to traditional halo orbits centered on the classical libration points, new orbits associated with artificial libration points are widely investigated. Baoyin and McInnes have investigated the periodic orbit around artificial Lagrange point in the Sun-Earth line [2] and periodic orbits displaced above or below the ecliptic plane of the Sun-Earth restricted three-body system [3]. Waters and McInnes studied periodic orbits around arbitrary artificial equilibrium [4]. These artificial equilibria have potential applications for future missions [5-7]. The Earth-Moon libration points have been a topic of great interest in recent years. The orbits around the collinear points are attractive because their unique positions are advantageous for several important applications in space mission design. In particular, periodic orbits displaced above the libration points in the Earth-Moon system are proposed for lunar far-side communications [8] If the orbit is visible from Earth, it can be used to provide communications between the Earth and the lunar poles. Simo and McInnes studied the dynamics and control of the periodic orbits above the $\mathrm{L}_{2}$ point [9]. The results indicated that such orbits cannot be maintained without active control due to their instability. A hybrid of solar sail and solar electric propulsion is proposed to maintain the spacecraft on the orbit [10]. However, the Moon is assumed to move in the Earth's orbital plane and the inclination of the Moon's orbit is not considered in previous studies. In this paper, the inclination of the Moon's orbital plane to the ecliptic plane is considered to model the dynamics of the solar sail. Furthermore, the sail area variation or hybrid propulsion is usually 
required to keep the solar sail on a reference displaced orbit. In this paper, a reflectivity device developed by JAXA is used for orbit control. JAXA developed this device for attitude control of IKAROS and future solar sail missions [11]. This device may provide an option for the orbit maintenance of the solar sail [12]. In this work, a solar sail including the reflectivity device is modeled and used to generate quasi-periodic and periodic orbits above the $\mathrm{L}_{2}$ libration point in the Earth-Moon system. The out-of-plane distance should be large enough to guarantee that both the lunar far-side and the equatorial regions of the Earth would be visible. Numerical analysis of stability and controllability of the orbits shows that the orbits are unstable but completely controllable with both the reflectivity rate and the sail attitude. A nonlinear sliding-mode control method is proposed to design the control law, leading to simpler tracking of the reference orbits. The results indicate that the solar sail may be maintained on the quasi-periodic orbit with only several percentage of the sail area equipped with the reflectivity device.

\section{Solar Sail Equipped with Reflectivity Control Devices}

Assume that part of the sail film is equipped with the Reflectivity Control Device (RCD) that can be switched between two different states. A part of the sunlight is specularly reflected for the state one, and the ratio of the reflected sunlight is defined by $\rho_{\mathrm{S}}$. The remaining sunlight is absorbed and the corresponding ratio is given by $1-\rho_{\mathrm{S}}$. The diffuse reflection is not considered in this paper since the amount of the sunlight of the diffuse reflection is very small for a smooth surface. Furthermore, the resultant force due to the reflected sunlight of the diffuse reflection is also very small because the incident sunlight is reflected from the surface at many angles, rather than at just one angle as in the case of specular reflection. It should be noted that all the sunlight is absorbed for the state two. The sail film is either in the state one or state two, and there is no intermediate state. Thus, the switch in state can be easily realized by turning the power ON and OFF.

Consider a solar sail of the total area $A$ and mass $m$. At some instant of time, the area $A_{1}$ is in state two and the remaining area in state one. The SRP acceleration exerted on the solar sail can be written as

$$
\mathbf{a}_{\mathrm{S}}=\mathbf{a}_{1}+\mathbf{a}_{2}
$$

The SRP accelerations $\mathbf{a}_{1}$ and $\mathbf{a}_{2}$ are due to sail area $\mathrm{A}_{1}$ and A- $\mathrm{A}_{1}$, respectively. 


$$
\begin{gathered}
\mathbf{a}_{1}=\frac{\mathrm{PA}}{\mathrm{m}}\left(\mathbf{r}_{\mathrm{S}} \cdot \mathbf{n}\right) \mathbf{r}_{\mathrm{S}} \\
\mathbf{a}_{2}=\frac{\mathrm{P}(\mathrm{A}-\mathrm{A})}{\mathrm{m}}\left[\left(1-\rho_{\mathrm{S}}\right)\left(\mathbf{r}_{\mathrm{S}} \cdot \mathbf{n}\right) \mathbf{r}_{\mathrm{S}}+2 \rho_{\mathrm{S}}\left(\mathbf{r}_{\mathrm{S}} \cdot \mathbf{n}\right)^{2} \mathbf{n}\right]
\end{gathered}
$$

The magnitude of the SRP force exerted on the sail is given by the sail characteristic acceleration $\kappa$, which is defined by the acceleration exerted on the solar sail when all RCDs are turned on and the sail normal is along the sunlight direction. It is assumed that $\kappa$ is constant over the scale of the problem. This acceleration depends directly on the total mass per unit area of the spacecraft. Then, the SRP acceleration may be rewritten as

$$
\mathbf{a}_{\mathrm{S}}=\frac{\kappa}{2}\left(\mathbf{r}_{\mathrm{S}} \cdot \mathbf{n}\right)\left[\left(1-\rho_{\mathrm{S}}+\mathrm{u} \rho_{\mathrm{S}}\right) \mathbf{r}_{\mathrm{S}}+2(1-\mathrm{u}) \rho_{\mathrm{S}}\left(\mathbf{r}_{\mathrm{S}} \cdot \mathbf{n}\right) \mathbf{n}\right]
$$

where $u$ is the ratio of the area in the state two divided by the total area, namely, $u=A_{1} / A$, which is denoted as the reflectivity rate.

Assume that the sail film includes small segments of the RCDs that can be actively controlled to switch between two states. Then, the reflectivity rate can vary continuously. If the area of the sail film equipped with the RCD is $A_{\max }, u$ can change from 0 to $A_{\max } / A$ continuously.

\section{Dynamical Equation}

Two references of frames are used to discuss the dynamics of the solar sail. The inertial frame OXYZ is defined as follows: the origin is at the mass center of the Earth. The X axis is directed along the intersection of the ecliptic plane and the Moon's orbital plane. The $\mathrm{Z}$ axis is perpendicular to the ecliptic plane, and the $\mathrm{Y}$ axis forms a right triad with the $\mathrm{X}$ and $\mathrm{Z}$ axes. The rotating frame oxyz is defined as follows: The origin is at the mass center of the Earth-Moon system. The $\mathrm{x}$ axis is directed from the Earth to the Moon. The $\mathrm{z}$ axis is perpendicular to the Moon's orbital plane, and the y axis forms a right triad with the $\mathrm{x}$ and $\mathrm{z}$ axes, as shown in Fig.1. 


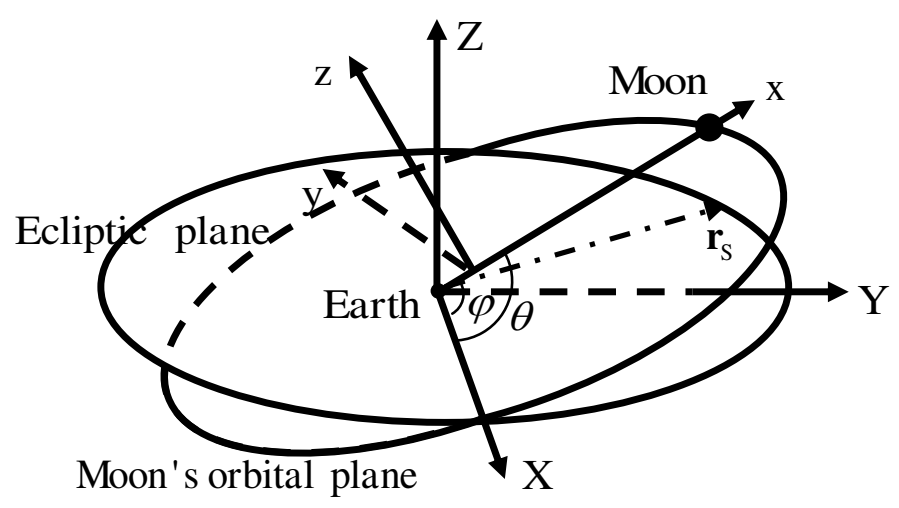

Figure 1 geometrical relationship of different planes.

The inclination of the Moon's orbital plane to the ecliptic plane $i$ varies between $4.950^{\circ}-5.317^{\circ}$ and the mean inclination is about $5.145^{\circ}$. In the Earth-Moon system, the Sun is considered moving through the ecliptic plane. The relative orientation of the inertial frame with respect to the rotating frame is described by two angles $(\theta, \mathrm{i})$ of the rotational sequence $\mathrm{R}_{2}(\theta) \leftarrow \mathrm{R}_{1}(\mathrm{i})$ (from the inertial frame to the rotating frame). The transition matrix can be given by

$$
\mathbf{A}=\mathbf{R}_{2}(\theta) \mathbf{R}_{1}(\mathrm{i})
$$

The phase angle $\theta$ is linearly proportional to the angular velocity of the Moon rotating around the Earth, namely, $\theta(\mathrm{t})=\theta\left(\mathrm{t}_{0}\right)+\omega_{\mathrm{M}}\left(\mathrm{t}-\mathrm{t}_{0}\right)$.

The sunlight lies in the plane of the ecliptic. The unit vector of the sunlight direction can be expressed in the inertial frame as

$$
\mathbf{r}_{\mathrm{SI}}=\left[\begin{array}{lll}
\cos \varphi & \sin \varphi & 0
\end{array}\right]^{\mathrm{T}}
$$

The phase angle $\varphi$ is linearly proportional to the angular velocity of the Earth rotating around the Sun, namely, $\varphi(\mathrm{t})=\varphi\left(\mathrm{t}_{0}\right)+\omega_{\mathrm{E}}\left(\mathrm{t}-\mathrm{t}_{0}\right)$.

The unit vector in the rotating frame can be expressed as

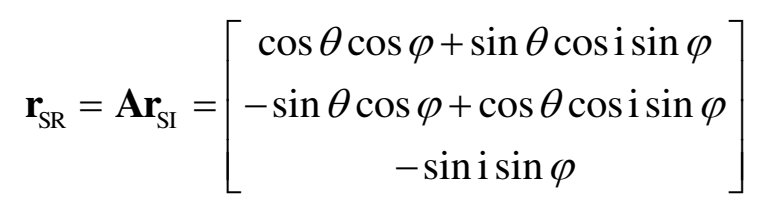

Another frame, $\mathrm{o}_{\mathrm{r}} \mathrm{x}_{\mathrm{r}} \mathrm{y}_{\mathrm{r}} \mathrm{z}_{\mathrm{r}}$, is used to describe the direction of the sail normal. The origin of the frame is at the mass center of the Earth; the $\mathrm{x}_{\mathrm{r}}$ axis is directed along the sunlight; the $\mathrm{z}_{\mathrm{r}}$ axis is directed along the $\mathrm{Z}$ axis, and the $\mathrm{y}_{\mathrm{r}}$ forms a right triad with the $\mathrm{x}_{\mathrm{r}}$ and $\mathrm{z}_{\mathrm{r}}$ axes. The pitch angle $\alpha$ and the clock angle $\gamma$ are used to describe the sail normal, where the pitch angle $\alpha$ is measured from the sunlight 
direction to the sail normal, and the clock angle $\gamma$ is measured from the $\mathrm{z}_{\mathrm{r}}$ axis to the projection line of the sail normal in the $y_{r} z_{r}$ plane. Then, the unit vector along the sail normal direction in the $o_{r} x_{r} y_{r}$ $\mathrm{z}_{\mathrm{r}}$ frame can be given by

$\mathbf{n}_{\mathrm{r}}=\left[\begin{array}{lll}\cos \alpha & \sin \alpha \sin \gamma & \sin \alpha \cos \gamma\end{array}\right]^{\mathrm{T}}$

It can be projected in the inertial frame as

$\mathbf{n}_{\mathrm{I}}=\left[\begin{array}{ccc}\cos \varphi & -\sin \varphi & 0 \\ \sin \varphi & \cos \varphi & 0 \\ 0 & 0 & 1\end{array}\right] \mathbf{n}_{\mathrm{r}}$

$=\left[\begin{array}{lll}\cos \varphi \cos \alpha-\sin \varphi \sin \alpha \sin \gamma & \sin \varphi \cos \alpha+\cos \varphi \sin \alpha \sin \gamma & \sin \alpha \cos \gamma\end{array}\right]^{\mathrm{T}}$

Similarly, the unit vector can be projected in the rotating frame as

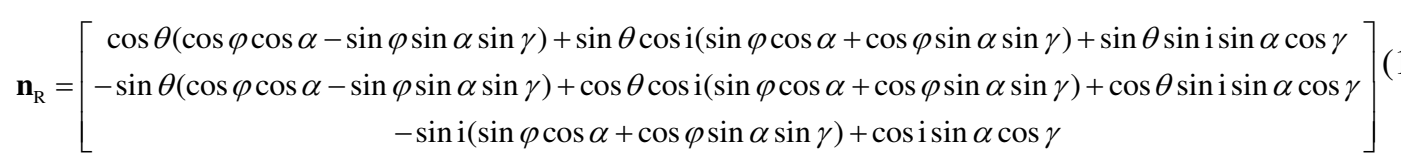

The motion of the solar sail is described in the rotating frame of constant angular velocity with the Earth-Moon in the circular orbit, as shown in Fig. 1. The units of the problem are chosen such that the gravitational constant, the Earth-Moon distances, the sum of the Earth and Moon mass, and so the angular velocity of the corotation are all unity. The vector equation of motion for the solar sail in the corotating frame may be written as

$$
\ddot{\mathbf{r}}+2 \omega_{\mathrm{M}} \times \dot{\mathbf{r}}+\nabla \mathrm{U}(\mathbf{r})=\mathbf{a}_{\mathrm{S}}
$$

where $U(\mathbf{r})=-\left[\frac{1}{2}\left|\omega_{\mathrm{M}} \times \mathbf{r}\right|^{2}+\frac{1-\mu}{\mathrm{r}_{1}}+\frac{\mu}{\mathrm{r}_{2}}\right]$.

The nondimensional equations of motion can be written as

$$
\left\{\begin{array}{l}
\ddot{x}-2 \dot{y}-x=-\frac{1-\mu}{r_{1}^{3}}(x+\mu)-\frac{\mu}{r_{2}^{3}}(x-1+\mu)+a_{x} \\
\ddot{y}+2 \dot{x}-y=-\frac{1-\mu}{r_{1}^{3}} y-\frac{\mu}{r_{2}^{3}} y+a_{y} \\
\ddot{z}=-\frac{1-\mu}{r_{1}^{3}} z-\frac{\mu}{r_{2}^{3}} z+a_{y}
\end{array}\right.
$$

The classical Lagrange points of the system can be obtained by letting $\nabla \mathbf{U}(\mathbf{r})=0$. The coordinate of the $\mathrm{L}_{2}$ Lagrange point is denoted as $\mathbf{r}_{\mathrm{L} 2}=\left[\begin{array}{lll}\mathrm{x}_{\mathrm{L} 2} & 0 & 0\end{array}\right]^{\mathrm{T}}$. Let a small displacement in the vicinity of the $\mathrm{L}_{2}$ Lagrange point be $\delta \mathbf{r}=\left[\begin{array}{lll}\xi & \eta & \zeta\end{array}\right]^{\mathrm{T}}$, such that $\mathbf{r}=\mathbf{r}_{\mathrm{L} 2}+\delta \mathbf{r}$. The dynamical 
equations for the solar sail near the $\mathrm{L}_{2}$ libration point can be rewritten as

$$
\left\{\begin{array}{l}
\left(\ddot{\mathrm{x}}_{\mathrm{L}_{2}}+\ddot{\xi}\right)-2 \dot{\eta}-\left(\mathrm{x}_{\mathrm{L}_{2}}+\xi\right)=-\frac{1-\mu}{\mathrm{r}_{1}^{3}}\left(\mathrm{x}_{\mathrm{L}_{2}}+\xi+\mu\right)-\frac{\mu}{\mathrm{r}_{2}^{3}}\left(\mathrm{x}_{\mathrm{L}_{2}}+\xi-1+\mu\right)+\mathrm{a}_{\mathrm{x}} \\
\ddot{\eta}+2\left(\dot{\mathrm{x}}_{\mathrm{L}_{2}}+\dot{\xi}\right)-\dot{\eta}=-\frac{1-\mu}{\mathrm{r}_{1}^{3}} \eta-\frac{\mu}{\mathrm{r}_{2}^{3}} \eta+\mathrm{a}_{\mathrm{y}} \\
\ddot{\zeta}=-\frac{1-\mu}{\mathrm{r}_{1}^{3}} \zeta-\frac{\mu}{\mathrm{r}_{2}^{3}} \zeta+\mathrm{a}_{\mathrm{z}}
\end{array}\right.
$$

where $\mathrm{r}_{1}=\sqrt{\left(\mathrm{x}_{\mathrm{L}_{2}}+\xi+\mu\right)^{2}+\eta^{2}+\zeta^{2}}, \mathrm{r}_{2}=\sqrt{\left(\mathrm{x}_{\mathrm{L}_{2}}+\xi-1+\mu\right)^{2}+\eta^{2}+\zeta^{2}}$.

Assume $\xi, \eta, \zeta$ are small, the equations of motion can be linearized by retaining only the first-order term in a Taylor series expansion. Since $\mathbf{r}_{\mathrm{L} 2}$ is an equilibrium point of Eq. (12) when there is no force exerted on the spacecraft, $\ddot{\mathrm{x}}_{\mathrm{L}_{2}}=0, \quad \dot{\mathrm{X}}_{\mathrm{L}_{2}}=0, \quad$ and $\mathrm{x}_{\mathrm{L} 2}=-\frac{1-\mu}{\left(\mathrm{x}_{\mathrm{L}_{2}}+\mu\right)^{1 / 2}}-\frac{\mu}{\left(\mathrm{x}_{\mathrm{L}_{2}}-1+\mu\right)^{1 / 2}}$ are used to simplify the linearized equation. In particular, the sail acceleration has been assumed constant under the small displacement from the L2 point. Therefore, the SRP acceleration is independent of the position of the solar sail. The linearized nondimensional equations of motion relative to the collinear libration point can be written as

$$
\left\{\begin{array}{l}
\ddot{\xi}-2 \dot{\eta}+\mathrm{U}_{\mathrm{xx}} \xi=\mathrm{a}_{\mathrm{x}} \\
\ddot{\eta}+2 \dot{\xi}+\mathrm{U}_{\mathrm{yy}} \eta=\mathrm{a}_{\mathrm{y}} \\
\ddot{\zeta}+\mathrm{U}_{\mathrm{zz}} \zeta=\mathrm{a}_{\mathrm{z}}
\end{array}\right.
$$

where $\mathrm{U}_{\mathrm{xx}}, \mathrm{U}_{\mathrm{yy}}, \mathrm{U}_{\mathrm{zz}}$ are the second-order derivatives of $\mathrm{U}$ with respect to $\mathrm{x}, \mathrm{y}, \mathrm{z}$, respectively, which can be given by

$$
\begin{aligned}
& \mathrm{U}_{\mathrm{xx}}=-2 \frac{1-\mu}{\left(\mathrm{x}_{\mathrm{L}_{2}}+\mu\right)^{3}}-\frac{2 \mu}{\left(\mathrm{x}_{\mathrm{L}_{2}}-1+\mu\right)^{3}}-1 \\
& \mathrm{U}_{\mathrm{yy}}=\frac{1-\mu}{\left(\mathrm{x}_{\mathrm{L}_{2}}+\mu\right)^{3}}+\frac{\mu}{\left(\mathrm{x}_{\mathrm{L}_{2}}-1+\mu\right)^{3}}-1 \\
& \mathrm{U}_{\mathrm{zz}}=\frac{1-\mu}{\left(\mathrm{x}_{\mathrm{L}_{2}}+\mu\right)^{3}}+\frac{\mu}{\left(\mathrm{x}_{\mathrm{L}_{2}}-1+\mu\right)^{3}}
\end{aligned}
$$

The linear dynamical equation is used to design the reference orbit, which is a solution of the differential equation. A particular solution is introduced since it is difficult to obtain the close form of the general solution. 


\section{Reference Orbit Design}

A simple method to design the reference orbit can be described as follows: first, the expression of the reference orbit is given and it is substituted into the differential equations (nonlinear or linearized equations). Thus, the differential equations become three algebraic equations, from which the attitude angles $\alpha, \gamma$, and the reflectivity rate $\mathrm{u}$ can be numerically solved. It should be noted that the numerical solution for the attitude angles and the reflectivity rate is time-varying. The variation ranges for these variables cannot be predicted since the process is numerically conducted. In engineering practice, the variations of these variables require the attitude control and the reflectivity rate control. Most importantly, the maximum reflectivity rate is restricted by the film area equipped with the reflectivity control device. Therefore, constant profiles of these variables are preferable. In addition, an explicit relation between the size of the reference orbit and the three variables is useful for the orbit design. The attitude angles and the reflectivity rate are assumed constant to design the reference orbit.

The reference orbit is bounded solution of Eq. (12). Then, the objective of the reference orbit design is to seek the desired bounded solution of Eq. (12). Under the assumption that the solar sail moves in the vicinity of the $\mathrm{L}_{2}$ libration point, the linearized equation (Eq. (14)) is employed for the reference orbit design since it is difficult to obtain the solution of the nonlinear differential equations.

\section{A. Quasi-periodic and periodic Solution}

The solution depends on the SRP acceleration, which is determined by the trigonometric functions of $\theta$ and $\varphi$ (time-dependent). It is difficult to derive the explicit solution without any simplification on the expression of the SRP acceleration. To simplify the expression, the relation "cosi=1" is used to describe the unit vectors of the sunlight and the sail normal direction. The assumption is reasonable since the inclination of the Moon's orbital plane $\mathrm{i}$ is very small $\left(4.950^{\circ}-5.317^{\circ}\right)$, cosi is very close to 1 (sini can be approximated by i). However, the expression cannot be further simplified by replacing sini with $i$. Thus, this approximation is used to solve the explicit solution. After simplification, the unit vectors of the sunlight direction and the sail normal direction may then be written approximately as

$$
\mathbf{r}_{\mathrm{SR}}=\left[\begin{array}{c}
\cos (\theta-\varphi) \\
-\sin (\theta-\varphi) \\
-\sin \mathrm{i} \sin \varphi
\end{array}\right]=\left[\begin{array}{c}
\cos \left(\omega_{\mathrm{M}} \mathrm{t}-\theta_{0}-\omega_{\mathrm{E}} \mathrm{t}+\varphi_{0}\right) \\
-\sin \left(\omega_{\mathrm{M}} \mathrm{t}-\theta_{0}-\omega_{\mathrm{E}} \mathrm{t}+\varphi_{0}\right) \\
-\sin \mathrm{i} \sin \varphi
\end{array}\right]=\left[\begin{array}{c}
\cos \left(\omega \mathrm{t}+\lambda_{0}\right) \\
-\sin \left(\omega \mathrm{t}+\lambda_{0}\right) \\
-\sin \mathrm{i} \sin \varphi
\end{array}\right]=\left[\begin{array}{c}
\cos \lambda \\
-\sin \lambda \\
-\sin \mathrm{i} \sin \varphi
\end{array}\right]
$$




$$
\mathbf{n}_{\mathrm{R}}=\left[\begin{array}{c}
\cos \lambda \cos \alpha+\sin \lambda \sin \alpha \sin \gamma+\sin \theta \sin \mathrm{i} \sin \alpha \cos \gamma \\
-\cos \alpha \sin \lambda+\cos \lambda \sin \alpha \sin \gamma+\cos \theta \sin \mathrm{i} \sin \alpha \cos \gamma \\
-\sin \mathrm{i}(\sin \varphi \cos \alpha+\cos \varphi \sin \alpha \sin \gamma)+\sin \alpha \cos \gamma
\end{array}\right]
$$

where $\omega$ is the difference between the Moon's angular velocity around the Earth and the Earth's angular velocity around the Sun. The expressions of the sunlight and the sail normal direction are substituted into the SRP acceleration to give

$$
\left\{\begin{array}{l}
\mathrm{a}_{\mathrm{x}}=\frac{\kappa}{2} \mathrm{u} \cos \alpha \cos \lambda+\kappa(1-\mathrm{u}) \cos ^{2} \alpha(\cos \lambda \cos \alpha+\sin \lambda \sin \alpha \sin \gamma+\sin \theta \sin \mathrm{i} \sin \alpha \cos \gamma) \\
\mathrm{a}_{\mathrm{y}}=-\frac{\kappa}{2} \mathrm{u} \cos \alpha \sin \lambda+\kappa(1-\mathrm{u}) \cos ^{2} \alpha(-\cos \alpha \sin \lambda+\cos \lambda \sin \alpha \sin \gamma+\cos \theta \sin \mathrm{i} \sin \alpha \cos \gamma) \\
\mathrm{a}_{\mathrm{z}}=-\frac{\kappa}{2} \mathrm{u} \cos \alpha \sin \mathrm{i} \sin \varphi+\kappa(1-\mathrm{u}) \cos ^{2} \alpha[-\sin \mathrm{i}(\sin \varphi \cos \alpha+\cos \varphi \sin \alpha \sin \gamma)+\sin \alpha \cos \gamma]
\end{array}\right.
$$

The expressions of the SRP acceleration (right-hand side of Eq. (14)) include three different frequencies, $\omega, \omega_{\mathrm{M}}, \omega_{\mathrm{E}}$. The z component of the SRP acceleration includes one time-dependent variable, $\varphi=\omega_{\mathrm{E}} \mathrm{t}+\varphi_{0}$. The general solution of the out-of-plane component can be written as

$$
\zeta(\mathrm{t})=\zeta_{0}+\zeta_{1} \sin \varphi+\zeta_{2} \cos \varphi
$$

Similarly, the SRP acceleration components include two time-dependent variables, $\theta=\omega_{\mathrm{M}} \mathrm{t}+\theta_{0}$ and $\lambda=\omega t+\lambda_{0}$. The general form of the solution for the in-plane motion may be written as

$$
\left\{\begin{array}{l}
\xi(\mathrm{t})=\xi_{0} \cos \lambda+\xi_{1} \sin \lambda+\xi_{2} \sin \theta \\
\eta(\mathrm{t})=\eta_{0} \sin \lambda+\eta_{1} \cos \lambda+\eta_{2} \cos \theta
\end{array}\right.
$$

The constant coefficients in Eqs. (18) and (19) are obtained by substituting the expressions into the linear dynamical equations. The $\mathrm{z}$ component of the linear equation can be given by

$$
\begin{aligned}
& -\zeta_{1} \omega_{\mathrm{E}}^{2} \sin \varphi-\zeta_{2} \omega_{\mathrm{E}}^{2} \cos \varphi+\mathrm{U}_{z z}\left(\zeta_{0}+\zeta_{1} \sin \varphi+\zeta_{2} \cos \varphi\right) \\
& =-\frac{\kappa}{2} \mathrm{u} \cos \alpha \sin \mathrm{i} \sin \varphi+\kappa(1-\mathrm{u}) \cos ^{2} \alpha[-\sin \mathrm{i}(\sin \varphi \cos \alpha+\cos \varphi \sin \alpha \sin \gamma)+\sin \alpha \cos \gamma]
\end{aligned}
$$

Let the coefficients of the trigonometric functions of both sides equal, the relations can be obtained as follows:

$$
\left\{\begin{array}{l}
\mathrm{U}_{\mathrm{zZ}} \zeta_{0}=\kappa(1-\mathrm{u}) \cos ^{2} \alpha \sin \alpha \cos \gamma \\
-\zeta_{1} \omega_{\mathrm{E}}^{2}+\mathrm{U}_{\mathrm{zz}} \zeta_{1}=-\frac{\kappa}{2} \mathrm{u} \cos \alpha \sin \mathrm{i}-\kappa(1-\mathrm{u}) \cos ^{3} \alpha \sin \mathrm{i} \\
-\zeta_{2} \omega_{\mathrm{E}}^{2}+\mathrm{U}_{\mathrm{zz}} \zeta_{2}=-\kappa(1-\mathrm{u}) \cos ^{2} \alpha \sin \alpha \sin \gamma \sin \mathrm{i}
\end{array}\right.
$$

Similarly, the remaining coefficients of the solution can be calculated by inserting the $\mathrm{x}$ and $\mathrm{y}$ 
components of the solution into the linear dynamical equations.

$$
\begin{aligned}
& \left\{\begin{array}{l}
-\xi_{0} \omega^{2}-2 \eta_{0} \omega+\mathrm{U}_{\mathrm{xx}} \xi_{0}=\frac{\kappa}{2} \mathrm{u} \cos \alpha+\kappa(1-\mathrm{u}) \cos ^{3} \alpha \\
-\xi_{1} \omega^{2}+2 \eta_{1} \omega+\mathrm{U}_{\mathrm{xx}} \xi_{1}=\kappa(1-\mathrm{u}) \cos ^{2} \alpha \sin \alpha \sin \gamma \\
-\xi_{2} \omega_{\mathrm{M}}^{2}+2 \eta_{2} \omega_{\mathrm{M}}+\mathrm{U}_{\mathrm{xx}} \xi_{2}=\kappa(1-\mathrm{u}) \cos ^{2} \alpha \sin \mathrm{i} \sin \alpha \cos \gamma
\end{array}\right. \\
& \left\{\begin{array}{l}
-\eta_{0} \omega^{2}-2 \xi_{0} \omega+\mathrm{U}_{\mathrm{yy}} \eta_{0}=-\frac{\kappa}{2} \mathrm{u} \cos \alpha-\kappa(1-\mathrm{u}) \cos ^{3} \alpha \\
-\eta_{1} \omega^{2}+2 \xi_{1} \omega+\mathrm{U}_{\mathrm{yy}} \eta_{1}=\kappa(1-\mathrm{u}) \cos ^{2} \alpha \sin \alpha \sin \gamma \\
-\eta_{2} \omega_{\mathrm{M}}^{2}+2 \xi_{2} \omega_{\mathrm{M}}+\mathrm{U}_{\mathrm{yy}} \eta_{2}=\kappa(1-\mathrm{u}) \cos ^{2} \alpha \sin \mathrm{i} \sin \alpha \cos \gamma
\end{array}\right.
\end{aligned}
$$

All coefficients of the solution depend on the sail attitude $\alpha, \gamma$, the sail characteristic acceleration $\kappa$ and the reflectivity rate $\mathrm{u}$. As the sail characteristic acceleration and reflectivity rate are given, the attitude angles will determine the solution. The maximum out-of-plane distance is determined by the coefficients of $\zeta$, which can be calculated from Eq. (21). The expression of $\zeta$ indicates that $\zeta_{1}$ and $\zeta_{2}$ are much smaller than $\zeta_{0}$ because $\mathrm{i}$ is a small number. Therefore, $\zeta_{0}$ provides a good approximation of the out-of-plane distance. The out-of-plane distance can be maximized by an optimal choice of the sail pitch and clock angles, determined by

$$
\gamma_{0}=0, \alpha_{0}=35.264^{\circ}
$$

The coefficients, $\xi_{1}, \eta_{1}, \zeta_{2}$, will be zero by inserting $\gamma_{0}=0$ into Eqs. (22) and (23). Thus, the solution for the out-of-plane displacement is simplified as

$$
\left\{\begin{array}{l}
\xi(\mathrm{t})=\xi_{0} \cos \lambda+\xi_{2} \sin \theta \\
\eta(\mathrm{t})=\eta_{0} \sin \lambda+\eta_{2} \cos \theta \\
\zeta(\mathrm{t})=\zeta_{0}+\zeta_{1} \sin \varphi
\end{array}\right.
$$

The coefficients, $\xi_{0}, \eta_{0}, \zeta_{0}, \xi_{2}, \eta_{2}, \zeta_{1}$, can be obtained by solving simultaneously Eqs. (22) and (23). The explicit expressions of these coefficients are given by

$$
\begin{gathered}
\xi_{0}=\frac{\omega^{2}+2 \omega-\mathrm{U}_{\mathrm{yy}}}{\left(-\mathrm{U}_{\mathrm{xx}} \mathrm{U}_{\mathrm{yy}}+\mathrm{U}_{\mathrm{yy}} \omega^{2}+\mathrm{U}_{\mathrm{xx}} \omega^{2}-\omega^{4}+4 \omega^{2}\right)}\left[\frac{\kappa}{2} \mathrm{u} \cos \alpha+\kappa(1-\mathrm{u}) \cos ^{3} \alpha\right] \\
\eta_{0}=\frac{\mathrm{U}_{\mathrm{xx}}-2 \omega-\omega^{2}}{\left(-\mathrm{U}_{\mathrm{xx}} \mathrm{U}_{\mathrm{yy}}+\mathrm{U}_{\mathrm{yy}} \omega^{2}+\mathrm{U}_{\mathrm{xx}} \omega^{2}-\omega^{4}+4 \omega^{2}\right)}\left[\frac{\kappa}{2} \mathrm{u} \cos \alpha+\kappa(1-\mathrm{u}) \cos ^{3} \alpha\right]
\end{gathered}
$$




$$
\begin{gathered}
\zeta_{0}=\frac{\kappa(1-\mathrm{u}) \cos ^{2} \alpha \sin \alpha}{\mathrm{U}_{\mathrm{zz}}} \\
\xi_{2}=\frac{\omega_{\mathrm{M}}^{2}+2 \omega_{\mathrm{M}}-\mathrm{U}_{\mathrm{yy}}}{\left(-\mathrm{U}_{\mathrm{xx}} \mathrm{U}_{\mathrm{yy}}+\mathrm{U}_{\mathrm{xx}} \omega_{\mathrm{M}}^{2}+\mathrm{U}_{\mathrm{yy}} \omega_{\mathrm{M}}^{2}-\omega_{\mathrm{M}}^{4}+4 \omega_{\mathrm{M}}^{2}\right)} \kappa(1-\mathrm{u}) \cos ^{2} \alpha \sin \alpha \sin \mathrm{i} \\
\eta_{2}=\frac{\omega_{\mathrm{M}}^{2}+2 \omega_{\mathrm{M}}-\mathrm{U}_{\mathrm{xx}}}{\left(-\mathrm{U}_{\mathrm{xx}} \mathrm{U}_{\mathrm{yy}}+\mathrm{U}_{\mathrm{xx}} \omega_{\mathrm{M}}^{2}+\mathrm{U}_{\mathrm{yy}} \omega_{\mathrm{M}}^{2}-\omega_{\mathrm{M}}^{4}+4 \omega_{\mathrm{M}}^{2}\right)} \kappa(1-\mathrm{u}) \cos ^{2} \alpha \sin \alpha \sin \mathrm{i} \\
\zeta_{1}=\frac{\mathrm{u}+2(1-\mathrm{u}) \cos ^{2} \alpha}{2\left(\omega_{\mathrm{E}}^{2}-\mathrm{U}_{\mathrm{zz}}\right)} \kappa \cos \alpha \sin \mathrm{i}
\end{gathered}
$$

The expressions indicate that two frequencies exist in the motion in xy-plane and a different frequency exists in z-direction. In addition, $\xi_{2}, \eta_{2}, \zeta_{1}$ are much smaller because they are proportional to sini. Therefore, the reference orbit is dominated by $\xi_{0}, \eta_{0}, \zeta_{0}$, which represents a periodic orbit displaced above the $\mathrm{L}_{2}$ libration point. The frequency of the periodic orbit is given by $\omega$, which is the difference between the Moon's orbital angular velocity around the Earth and the Earth's orbital angular velocity around the Sun. The motion induced by $\xi_{2}, \eta_{2}, \zeta_{1}$ will introduce slight fluctuations superimposed on the periodic motion.

If the inclination of the Moon's orbital plane is set to zero ( $i=0)$, the result will degenerate to the periodic solution discussed by Simo and McInnes [8] and McInnes [13], where the inclination of the Moon's orbital plane is not considered. In this case, the periodic solution can be chosen as

$$
\left\{\begin{array}{l}
\xi(\mathrm{t})=\xi_{0} \cos \lambda \\
\eta(\mathrm{t})=\eta_{0} \sin \lambda \\
\zeta(\mathrm{t})=\zeta_{0}
\end{array}\right.
$$

The analysis indicates that the maximum out-of-plane displacements of quasi-periodic and periodic orbits are similar. It is determined by the characteristic acceleration and the reflectivity rate of the solar sail. The maximum value can be obtained by substituting $\gamma_{0}=0, \alpha_{0}=35.264^{\circ}$, and $\mathrm{U}_{\mathrm{zz}}=3.1904$ into Eq. (28):

$$
\zeta_{\max }=0.12064 \kappa(1-\mathrm{u})
$$

The maximum out-of-plane displacement is linearly proportional to the characteristic acceleration. 


\section{B. Error Analysis}

The quasi-periodic and periodic orbits are constructed based on the linear dynamical equation. The linearization will take into account the system model errors. Furthermore, the design process of the quasi-periodic orbit and periodic orbit assumes that the inclination of the lunar orbit is small (or equal to zero), which will also induce some errors. Therefore, the designed SRP acceleration cannot completely cancel the acceleration induced by the nonlinear dynamics. It is important to verify that the system model error is very small compared to the nominal SRP acceleration. For a given reference orbit, the corresponding attitude angles and reflectivity rate can be obtained according to the above design process. Thus, the nominal SRP acceleration corresponding to the reference orbit, $\mathbf{a}_{\mathrm{SR}}$, can be calculated from Eq. (4). The acceleration induced by the nonlinear dynamics is given by

$$
\mathbf{a}_{\mathrm{ND}}=\ddot{\mathbf{r}}_{\mathrm{ref}}+2 \boldsymbol{\omega}_{\mathrm{M}} \times \dot{\mathbf{r}}_{\mathrm{ref}}+\nabla \mathrm{U}\left(\mathbf{r}_{\mathrm{ref}}\right)
$$

Then, the absolute and relative errors between $\mathbf{a}_{\mathrm{SR}}$ and $\mathbf{a}_{\mathrm{ND}}$ are defined as

$$
\begin{gathered}
\mathbf{a}_{\mathrm{AE}}=\mathbf{a}_{\mathrm{SR}}-\mathbf{a}_{\mathrm{ND}} \\
\mathrm{a}_{\mathrm{RE}}=\frac{\left|\mathbf{a}_{\mathrm{SR}}-\mathbf{a}_{\mathrm{ND}}\right|}{\left|\mathbf{a}_{\mathrm{SR}}\right|}
\end{gathered}
$$

Given the solar sail parameters, the coefficients of the solutions associated to the quasi-periodic and periodic orbits can be calculated from Eqs. (21) to (23). Table 1 gives the parameters of two examples, one for a quasi-periodic orbit and the other for a periodic orbit above the $\mathrm{L}_{2}$ point in the Earth-Moon system. Figure 3 shows the relative and absolute errors, where the absolute and relative errors are less than $4.1 \times 10^{-4}$ for a periodic orbit and $6.1 \%$ for a quasi-periodic orbit. The errors almost double for the periodic orbit since the assumption of $i=0$ introduces extra errors compared to the quasi-periodic case. The solar sail cannot stay on the reference orbit even it is placed exactly on the orbit due to the system model errors and the instability of the relative motion in the vicinity of the $\mathrm{L}_{2}$ point. Therefore, active control is necessary to maintain the solar sail on the orbit. 
Table 1 Parameters of quasi-periodic and periodic orbits

\begin{tabular}{|c|c|c|c|c|c|c|c|}
\hline & $\alpha\left(^{\circ}\right)$ & $\gamma\left({ }^{\circ}\right)$ & $\kappa$ & $\mathrm{u}$ & $\Xi$ & $\eta$ & $\zeta$ \\
\hline Quasi-periodic & 35.26 & 0 & 0.01 & 0.05 & $\begin{array}{l}\xi_{0}=1.9239 \times 10^{-4} \\
\xi_{1}=0 \\
\xi_{2}=1.8994 \times 10^{-5}\end{array}$ & $\begin{array}{l}\eta_{0}=-3.7613 \times 10^{-3} \\
\eta_{1}=0 \\
\eta_{2}=2.4356 \times 10^{-4}\end{array}$ & $\begin{array}{l}\zeta_{0}=1.1461 \times 10^{-3} \\
\zeta_{1}=-1.5136 \times 10^{-4} \\
\zeta_{2}=0\end{array}$ \\
\hline Periodic & 35.26 & 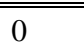 & 0.01 & 0.15 & $1.8757 \times 10^{-4}$ & $-3.6663 \times 10^{-3}$ & $1.0255 \times 10^{-3}$ \\
\hline
\end{tabular}
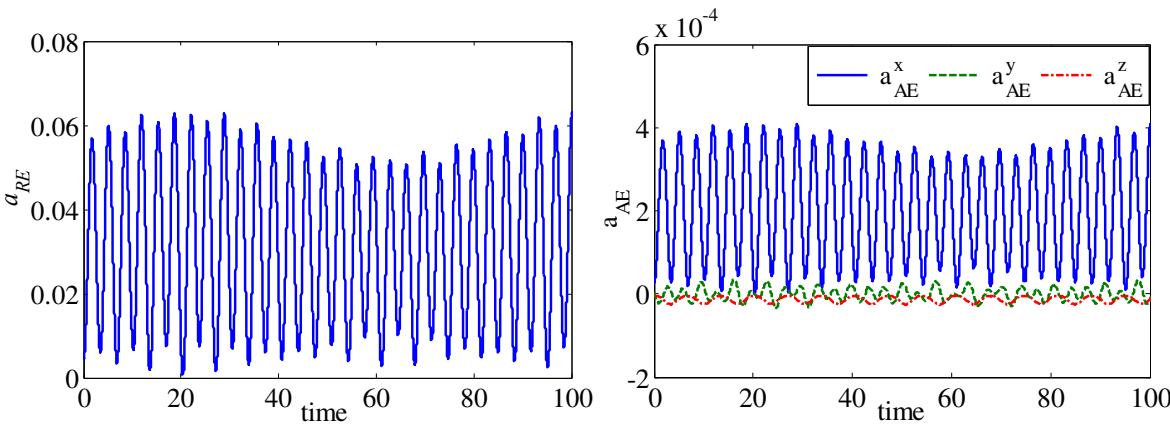

(a) relative error of the quasi-periodic orbit

(b) absolute error of the quasi-periodic orbit

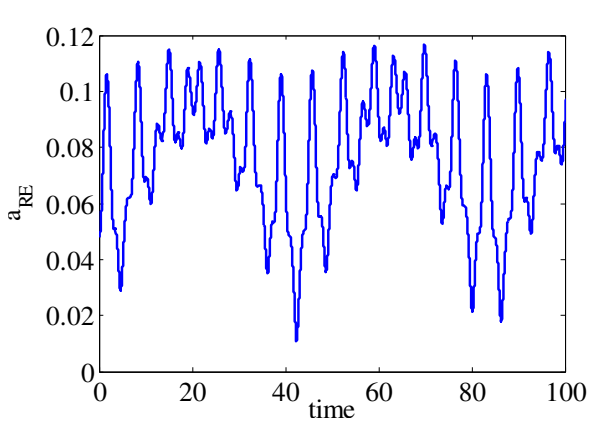

(c) relative error of the periodic orbit

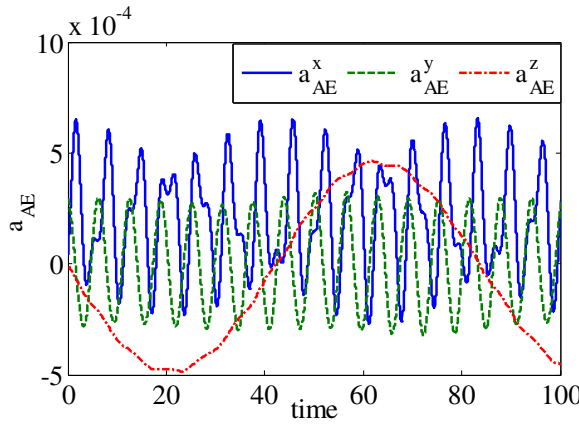

(d) absolute error of the periodic orbit

Figure 2 Relative and absolute errors for two reference orbits

\section{Nonlinear Sliding-mode Control}

The sliding mode control is one of the most important approaches dealing with model uncertainty.

An active control is required to keep the solar sail in the vicinity of the Libration point. Modeling errors have strong adverse effects on nonlinear control systems. Also, the sliding mode controller design provides a systematic approach to the problem of maintaining stability and consistent performance in the case of modeling imprecision [14]. A sliding surface should be defined and the control will force the trajectory to converge to the sliding surface when the system trajectory has reached the surface. Ideally, once intercepted, the switched control maintains the trajectory on the surface for subsequent time. The sliding surface is defined as

$$
\mathbf{S}=\mathbf{r}-\mathbf{r}_{\text {ref }}+\Gamma\left(\dot{\mathbf{r}}-\dot{\mathbf{r}}_{\text {ref }}\right)
$$

The reference trajectory has been defined as the periodic or quasi-periodic orbit in the above section. 
In the rotating frame, the corresponding position, velocity and acceleration of the reference trajectory can be given by

$$
\mathbf{r}_{\text {ref }}=\left[\begin{array}{c}
\mathrm{x}_{\mathrm{L}_{2}}+\xi(\mathrm{t}) \\
\eta(\mathrm{t}) \\
\zeta(\mathrm{t})
\end{array}\right], \quad \dot{\mathbf{r}}_{\mathrm{ref}}=\left[\begin{array}{c}
\dot{\xi}(\mathrm{t}) \\
\dot{\eta}(\mathrm{t}) \\
\dot{\zeta}(\mathrm{t})
\end{array}\right], \quad \ddot{\mathbf{r}}_{\text {ref }}=\left[\begin{array}{c}
\ddot{\xi}(\mathrm{t}) \\
\ddot{\eta}(\mathrm{t}) \\
\ddot{\zeta}(\mathrm{t})
\end{array}\right]
$$

Lyapunov method is used to determine the stability of the system. A generalized Lyapunov function, which characterizes the motion of the state trajectory to the sliding surface, is defined in terms of the sliding surface. For each chosen switched control structure, the control law is designed so that the derivative of the Lyapunov function is negative definite, thus guaranteeing the motion of the state trajectory to the surface. The Lyapunov function is chosen to be

$$
\mathrm{V}=\frac{1}{2} \mathbf{S}^{\mathrm{T}} \mathbf{S}
$$

The derivative of the Lyapunov function can be given by

$$
\begin{aligned}
& \dot{\mathrm{V}}=\mathbf{S}^{\mathrm{T}} \dot{\mathbf{S}}=\mathbf{S}^{\mathrm{T}}\left[\left(\dot{\mathbf{r}}-\dot{\mathbf{r}}_{\text {ref }}\right)+\Gamma\left(\ddot{\mathbf{r}}-\ddot{\mathbf{r}}_{\text {ref }}\right)\right] \\
& =\mathbf{S}^{\mathrm{T}}\left[\left(\dot{\mathbf{r}}-\dot{\mathbf{r}}_{\text {ref }}\right)+\Gamma\left(\mathbf{a}_{\mathrm{s}}-2 \omega_{\mathrm{M}} \times \dot{\mathbf{r}}-\nabla \mathrm{U}(\mathbf{r})-\ddot{\mathbf{r}}_{\text {ref }}\right)\right]
\end{aligned}
$$

The SRP acceleration is used to stabilize the system. To guarantee the negativity of the derivative, the solar radiation acceleration is designed as

$$
\mathbf{a}_{\mathrm{S}}=2 \omega_{\mathrm{M}} \times \dot{\mathbf{r}}+\nabla \mathrm{U}(\mathbf{r})+\ddot{\mathbf{r}}_{\mathrm{ref}}-\Gamma^{-1}\left[\sigma \operatorname{sign}(\mathbf{S})+\left(\dot{\mathbf{r}}-\dot{\mathbf{r}}_{\mathrm{ref}}\right)\right]
$$

Then, the derivative of the Lyapunov function can be rewritten as

$$
\dot{\mathrm{V}}=-\mathbf{S}^{\mathrm{T}} \sigma \operatorname{sign}(\mathbf{S})
$$

Thus, the derivative of the Lyapunov function is negative and the system is asymptotically stable. The required SRP acceleration for the stabilizing system is given by Eq. (41), which is achieved by adjusting the pitch and clock angles of the sail attitude, with respect to the sunlight and the reflectivity rate of the sail film. Therefore, the actual control variables are the attitude angles and the reflectivity rate. However, the explicit expressions of the control variables cannot be given since the SRP acceleration is a nonlinear function of the three parameters. The next step is to calculate the control variables for a given control acceleration.

The required SRP acceleration for a quasi-periodic solution of the nonlinear dynamical 
equation can be given by

$$
\mathbf{a}_{\mathrm{S} 0}=2 \omega_{\mathrm{M}} \times \dot{\mathbf{r}}_{\mathrm{ref}}+\nabla \mathrm{U}\left(\mathbf{r}_{\mathrm{ref}}\right)+\ddot{\mathbf{r}}_{\mathrm{ref}}
$$

The reference control variables are denoted as $\alpha_{0}(\mathrm{t}), \gamma_{0}(\mathrm{t}), \mathrm{u}(\mathrm{t})$. Assume that the errors of the sail relative to the reference orbit are small. Thus, the control variables are also in the vicinity of the reference values. The SRP acceleration can be linearized as

$$
\mathbf{a}_{\mathrm{S}}=\mathbf{a}_{\mathrm{S} 0}+\left.\frac{\partial \mathbf{a}_{\mathrm{S}}}{\partial \alpha}\right|_{\substack{\alpha=\alpha_{0} \\ \gamma=\gamma_{0} \\ \mathrm{u}=\mathrm{u}_{0}}} \delta \alpha+\left.\frac{\partial \mathbf{a}_{\mathrm{S}}}{\partial \gamma}\right|_{\substack{\alpha=\alpha_{0} \\ \gamma=\gamma_{0} \\ \mathrm{u}=\mathbf{u}_{0}}} \delta \gamma+\left.\frac{\partial \mathbf{a}_{\mathrm{S}}}{\partial \mathrm{u}}\right|_{\substack{\alpha=\alpha_{0} \\ \gamma=\gamma_{0} \\ \mathrm{u}=\mathrm{u}_{0}}} \delta \mathrm{u}
$$

The control law can be represented by the differences from the reference values of the control variables given by

$$
\left[\begin{array}{l}
\delta \alpha \\
\delta \gamma \\
\delta \mathrm{u}
\end{array}\right]=\left[\begin{array}{lll}
\frac{\partial \mathbf{a}_{\mathrm{s}}}{\partial \alpha} & \frac{\partial \mathbf{a}_{\mathrm{s}}}{\partial \gamma} & \frac{\partial \mathbf{a}_{\mathrm{s}}}{\partial \mathrm{u}}
\end{array}\right]^{-1}\left(\mathbf{a}_{\mathrm{s}}-\mathbf{a}_{\mathrm{s} 0}\right)
$$

For a quasi-periodic reference orbit, the solution associated to the linearized equation can provide a reasonable control law. For a periodic reference orbit, the control law may make the system unstable due to the error model in $\mathbf{a}_{\mathrm{s} 0}$. In this case, the nonlinear Eq. (44) is used to obtain the control law. The explicit solution of Eq. (44) cannot be obtained analytically. With the solution of Eq. (45) as the initial guess, the accurate numerical solution of Eq. (44) can be obtained by using a simple Newton iteration process.

\section{Numerical Simulations}

In the following simulations, the nonlinear dynamical equation is used for the numerical integration. The geometrical relationship is employed to calculate the sunlight direction [Eqs. (7) and (10)]. The control law is generated by solving the nonlinear algebraic equation defined by Eq. (44). A quasi-periodic orbit and a periodic orbit above the $\mathrm{L}_{2}$ libration point in the Earth-Moon system are expressed as reference orbits. The simulation parameters are given in Table 2. The maximum displacements of the periodic and quasi-periodic orbits are 0.0060769 and 0.0065171 , respectively. This allows the spacecraft to view both the lunar far-side and the equatorial regions of the Earth. The reflectivity rate for the reference orbits are selected to guarantee that the control law is achievable. Also, there is no negative value for the reflectivity rate appearing in the control law. The initial position errors of the spacecraft with respect to the reference orbits are 1e-4 nondimensional unit in 
$\mathrm{x}, \mathrm{y}$, and $\mathrm{z}$ directions.

Table 2 parameters for simulation

\begin{tabular}{|c|c|c|c|c|c|c|}
\hline & $\Gamma$ & $\sigma$ & $\alpha\left(^{\circ}\right)$ & $\gamma\left({ }^{\circ}\right)$ & $\kappa$ & $\mathrm{u}$ \\
\hline Quasi -periodic & $\operatorname{diag}([50,50,50])$ & $\operatorname{diag}([2 \mathrm{e}-4,2 \mathrm{e}-4,2 \mathrm{e}-4])$ & 35.26 & 0 & 0.073 & 0.26 \\
\hline Periodic & $\operatorname{diag}([10000,10000,10000])$ & $\operatorname{diag}([0.01,0.01,0.01])$ & 35.26 & 0 & 0.068 & 0.31 \\
\hline
\end{tabular}

Figure 3 gives the controlled orbit in the Earth-Moon rotating frame for the two cases, respectively.

Figure 4 gives the time histories of the control variables for the two cases, respectively. For the nonlinear dynamical equation, a periodic or quasi-periodic solution does not exist if the attitude and the reflectivity rate remain constant. For each point on the reference orbit, the pitch angle, the clock angle and reflectivity rate [Eq. (11)] can be solved to satisfy the nonlinear dynamical equation. The reference values of the attitude and reflectivity parameters based on the linear dynamical equation provide very good approximations for the solution of the nonlinear dynamical equation. The results show that the control variables converge to the solution of the nonlinear dynamical equation when the controlled orbits converge to the reference orbit.

For the quasi-periodic orbit, the maximum pitch and clock angles are a little less than those of the periodic orbit. This means that the cost for the attitude control is more for the periodic orbit. In addition, the reflectivity parameter of the quasi-periodic orbit is less than 0.26 , whereas it is about 0.44 for the periodic orbit. This means that more reflectivity control devices are required to keep the solar sail in the periodic orbit. For a given solar sail, the quasi-periodic orbit is advantageous over the periodic orbit in maximum out-of-plane displacement and reflectivity control device requirement. 


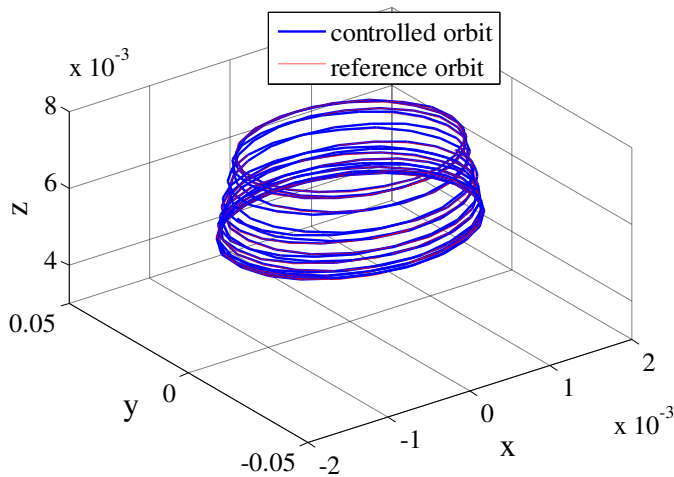

(a) quasi-periodic orbit

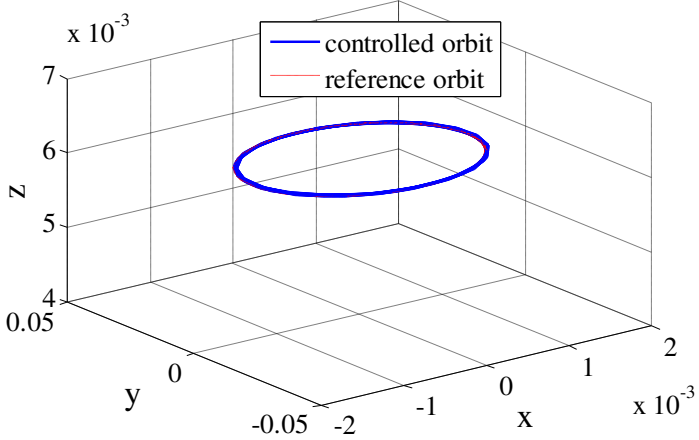

(b) periodic orbit

Figure 3 Controlled orbits above $\mathrm{L}_{2}$ libration point in Earth-Moon system
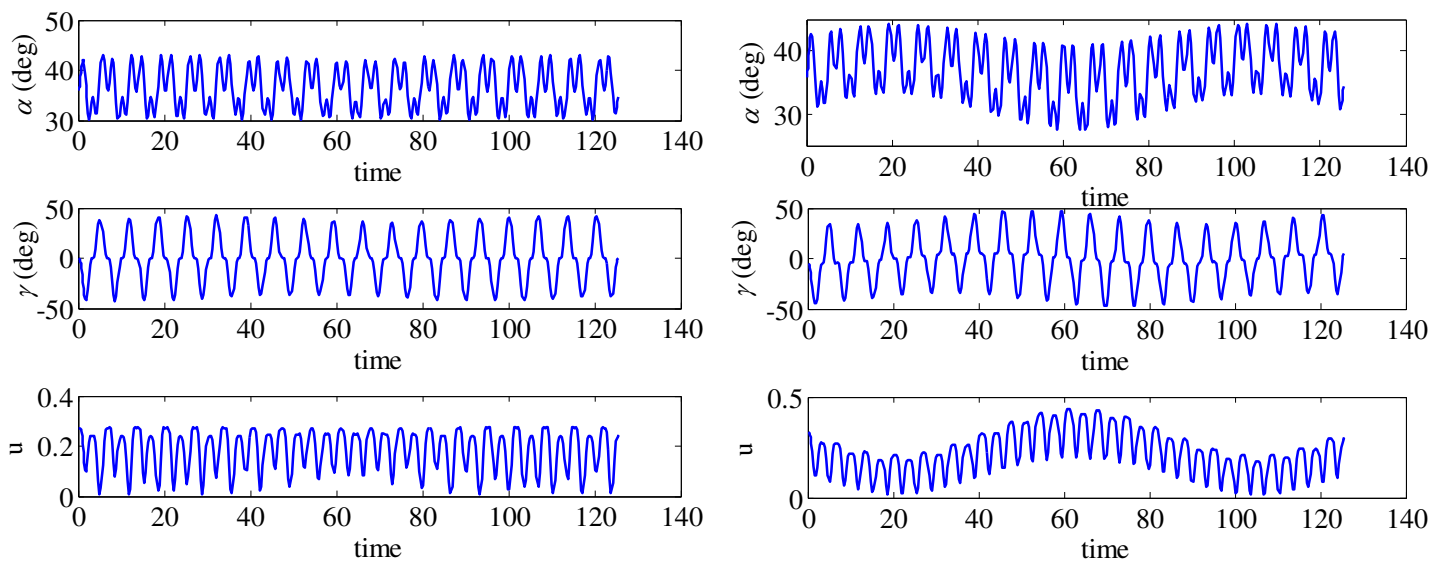

(a) quasi-periodic orbit

(b) periodic orbit

Figure 4 time histories of the control variables

\section{Conclusion}

Based on the assumption that the inclination of the lunar orbit to the ecliptic plane is small, a new family of new quasi-periodic orbits above the libration point in the Earth-Moon system is proposed for lunar far-side telecommunications. This family of orbits is closer to the natural solution of the nonlinear dynamical equation than the periodic orbits. Less effort is required to maintain the solar sail on the quasi-periodic orbit than on the periodic orbit. Therefore, the quasi-periodic orbit is advantageous over the periodic orbit with respect to the control effort. 


\section{Acknowledgment}

This work has been supported by the National Natural Science Foundation of China (11272004) and China' civil space funding.

\section{References}

[1] McInnes, C. R., McDonald, A. J., Simmons, J. F. L., MacDonald, E. W., "Solar Sail Parking in Restricted Three-Body Systems," Journal of Guidance, Control, and Dynamics, Vol. 17, No.2, 1994, pp. 399-406. DOI: 10.2514/3.21211

[2] Baoyin, H., and McInnes, C., "Solar Sail Halo Orbits at the Sun-Earth Artificial L1 Point," Celestial Mechanics and Dynamical Astronomy, Vol. 94, No. 2, 2006, pp. 155-171. DOI: 10.1007/s10569-005-4626-3

[3] Baoyin, H., and McInnes, C, R., "Solar Sail Orbits at Artificial Sun-Earth Libration Points," Journal of Guidance, Control, and Dynamics, Vol. 28, No. 6, 2005, pp.1328-1330. DOI: $10.2514 / 1.14598$

[4] Waters, T., and McInnes, C., "Periodic Orbits Above the Ecliptic in the Solar-Sail Restricted Three-Body Problem," Journal of Guidance, Control, and Dynamics, Vol. 30, No. 3, 2007, pp. 687-693. DOI: 10.2514/1.26232

[5] Farquhar, R., and Kamel, A., "Quasi-Periodic Orbits about the Translunar Libration Point," Celestial Mechanics, Vol. 7, 1973, pp. 458-473. DOI: 10.1007/BF01227511

[6] Howell, K., and Marchand, B., "Natural and Non-Natural Spacecraft Formations Near L1 and L2 Libration Points in the Sun-Earth/Moon Ephemerics System," Dynamical Systems: An International Journal, Vol. 20, No. 1, 2005, pp. 149-173. DOI:10.1080/1468936042000298224

[7] Grebow, D., Ozimek, M., Howell, K., "Multi-Body Orbit Architectures for Lunar South Pole Coverage," Advances in the Astronautical Science, Vol. 124, No. 2, 2006, pp. 1285-307.

[8] Simo, J., and McInnes, C. R., "Displaced Solar Sail Orbits: Dynamics and Applications," Advances in the Astronautical Science, Vol. 136, 2010, pp. 1803-1816.

[9] Simo, J., and McInnes, C. R., "Asymptotic Analysis of Displaced Lunar Orbits," Journal of Guidance,Control and Dynamics, Vol. 32, No. 5, 2009, pp. 1666-1671. DOI: 10.2514/1.43703

[10] Simo, J., and McInnes, C. R., "Designing Displaced Lunar Orbits Using Low-Thrust Propulsion," Journal of Guidance, Control and Dynamics, Vol. 33, No. 1, 2010, pp.259-265. DOI: $10.2514 / 1.45305$

[11] Tsuda, Y., Mori, O., Funase, R., Sawada, H., Yamamoto, T., Saiki, T., Endo, T., Kawaguchi J., "Flight Status of IKAROS Deep Space Solar Sail Demonstrator," Acta Astronautica Vol. 69, Issues 9-10. 2011, pp. 833-840. DOI: 10.1016/j.actaastro.2011.06.005 
[12] Mimasu, Y., Yamaguchi, T., Matsumoto, M., Nakamiya, M., Funase, R., Kawaguchi, J., "Spinning Solar Sail Orbit Steering via Spin Rate Control," Advances in Space Research, Vol. 48, Iss. 11, 2011, pp.1810-1821. DOI: 10.1016/j.asr.2011.08.030

[13] McInnes, C. R., "Solar Sail Trajectories at the Lunar $\mathrm{L}_{2}$ Lagrange Point," Journal of Spacecraft and Rocket, Vol.30, No.6, 1993, pp.782-784. DOI: 10.2514/3.26393

[14] Jovan, D., Sai-Ming, L., Raman, K., "Robust Adaptive Variable Structure Control of Spacecraft Under Control Input Saturation," Journal of Guidance,Control,and Dynamics, Vol. 24, No.1 ,2001,pp.14-22. DOI: 10.2514/2.4704 